\title{
Concomitant fenestration and phlebectasia of the right internal jugular vein: a unique finding in anatomical dissection
}

\author{
E. Cvetko, M. Meznarič \\ Institute of Anatomy, Faculty of Medicine, University of Ljubljana, Ljubljana, Slovenia
}

[Received: 2 November 2016; Accepted: 4 January 2017]

\begin{abstract}
The internal jugular vein (IJV) is an important vascular structure for oncologists and radiologists and is also a frequently used central venous route. The variations in the pattern of its course, and knowledge of its variations, are important. During the anatomical dissection of a 70-year-old male cadaver, fenestration involving a short segment $(2.5 \mathrm{~cm})$ of the right IJV in the superior part of the carotid triangle and a large dilatation (phlebectasia) of the $I J V$, involving its nonfenestrated segment were found - a case that has not previously been reported. The aetiology and clinical implications of the concurrent anomalies are described. Clinicians and surgeons performing neck vascular or reconstructive surgery should be made aware of both IJV variations in order to prevent inadvertent injury and avoid invasive investigations and inappropriate treatment. (Folia Morphol 2017; 76, 3: 519-522)
\end{abstract}

Key words: internal jugular vein, anatomical variation, fenestration, phlebectasia, dilatation

\section{INTRODUCTION}

The internal jugular vein (IJV) is the largest vein in the neck and drains the venous blood from the cranium, the facial region and the neck. The IJV is a significant landmark that is encountered during dissection of the cervical lymph nodes in oncological surgery, central venous catheter insertion and interventional radiological procedures; familiarity with its probable anatomical variations is therefore crucial $[4,9]$. Divisions (duplication or fenestration) of the IJV are rare and are mostly observed during neck surgery and diagnostic imaging, and only very rarely during cadaver dissection $[3,4,6]$. Phlebectasia denotes an abnormal saccular or fusiform dilated segment of the vein. Other terms used to describe this condition are venous aneurysm, venous cyst, aneurysmal varix and venectasia [7]. Internal jugular phlebectasia is rare, with reports limited to clinical cases alone. The concomitant occurrence of the IJV division and phlebectasia could only be found in two prior reports of clinical cases $[8,9]$ and were revealed during diagnostic imaging. Both cases reported phlebectasia of the fenestrated segment of the IJV. In the case presented, the phlebectasia involved nonfenestrated segment of the IJV, an entity that has not yet been reported.

\section{CASE REPORT}

During a course on the subject of applied clinical anatomy for dental students and the dissection of the neck of a 70-year-old male embalmed cadaver, fen- 


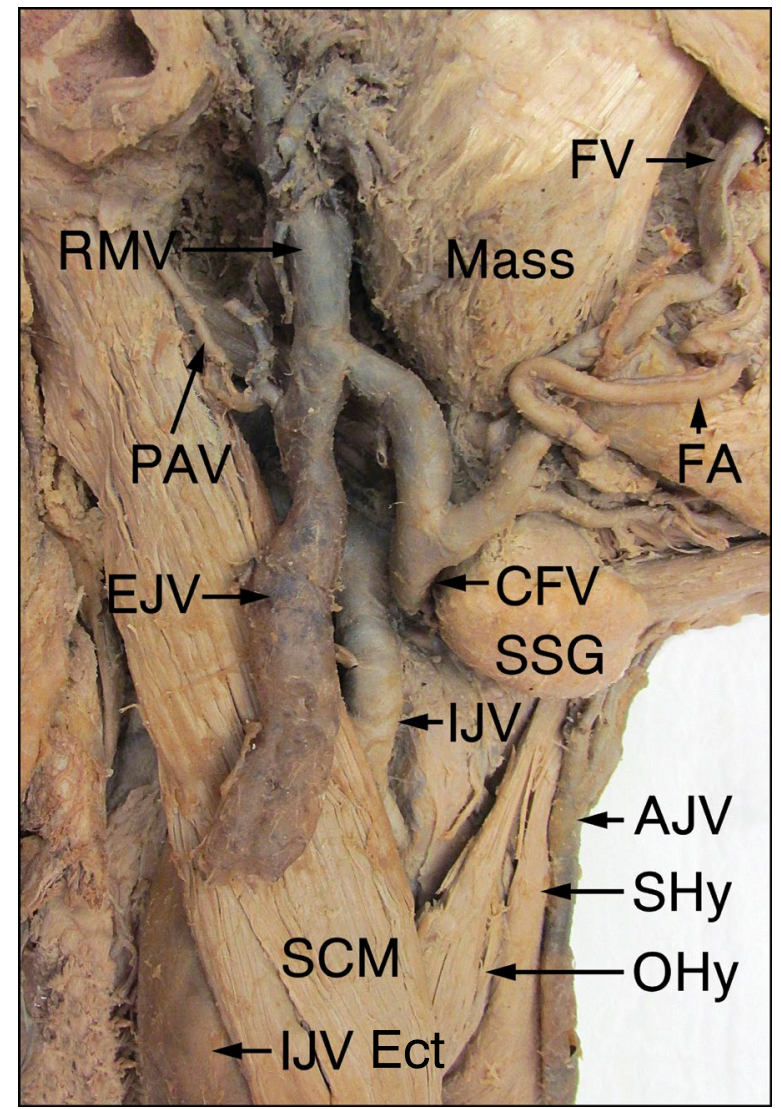

Figure 1. Superficial dissection of the right side of the neck, showing the medial branch of the fenestrated internal jugular vein (IJV) in the carotid triangle and internal jugular phlebectasia (Ect) in the lateral cervical triangle; FV — facial vein; RMV — retromandibular vein; Mass - masseter muscle; PAV — posterior auricular vein; FA — facial artery; EJV — external jugular vein (cut); CFV — common facial vein; $\mathrm{M}$ - medial branch of fenestrated IJV; SSG — submandibular salivary gland; AJV — anterior jugular vein; SHy — sternohyoid muscle; SCM — sternocleidomastoid muscle; $\mathrm{OHy}$ — omohyoid muscle, IJV Ect — internal jugular phlebectasia.

estration and phlebectasia of the IJV were observed on its right side (Figs. 1, 2). The IJV originated at the jugular foramen as a single vessel, descended parallel to the carotid arteries and entered the subclavian vein behind the sternoclavicular joint. The IJV bifurcated into the medial and lateral branches, just beneath the posterior belly of the digastric muscle, and reunited again at the level of the superior border of the thyroid cartilage. The fenestrated segment of the IJV was $2.5 \mathrm{~cm}$ in length and had a wide window $(1.5 \mathrm{~cm})$ with no structure passing through. The lateral branch of the fenestrated IJV was slightly larger in diameter $(4 \mathrm{~mm})$ than the medial branch $(3 \mathrm{~mm})$. The upper portion of the medial branch received the common facial vein. The lateral branch had no tributaries. Below the re-

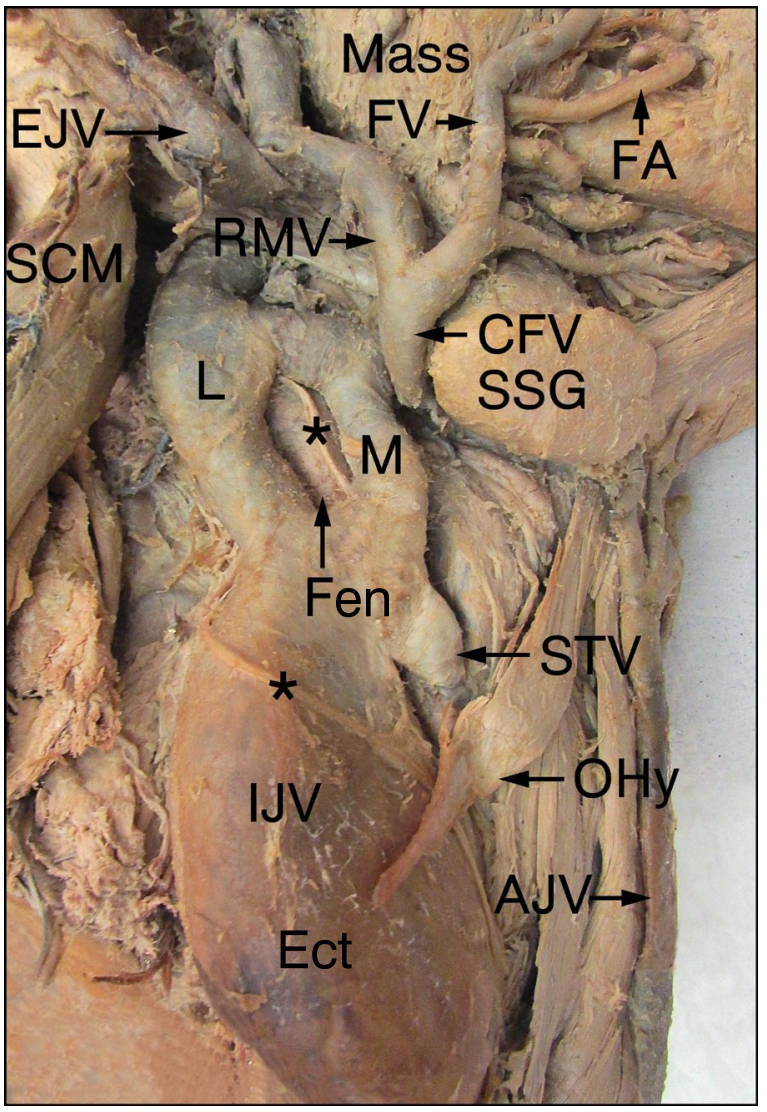

Figure 2. Deep dissection of the right side of the neck showing the fenestration (Fen) and phlebectasia (Ect) of the internal jugular vein (IJV); Mass - masseter muscle; EJV — external jugular vein (retracted); FV — facial vein; FA — facial artery; SCM sternocleidomastoid muscle (retracted); RMV — retromandibular vein; CFV — common facial vein; SSG — submandibular salivary gland; $\mathrm{L}$ - lateral branch of fenestrated IJV; $\mathrm{M}$ - medial branch of fenestrated IJV; STV — superior thyroid vein; $\mathrm{OHy}$ — omohyoid muscle; AJV — anterior jugular vein; asterisk — superior ramus of the ansa cervicalis.

union of the medial and lateral branches the IJV continued downward with a large phlebectasia (4 cm in diameter, $7 \mathrm{~cm}$ in length). The IJV narrowed to a diameter of $2 \mathrm{~cm}$ just before joining the subclavian vein. The superior thyroid vein emptied into the superior part of the internal jugular phlebectasia.

The superior ramus of the ansa cervicalis passed behind the IJV fenestration and the basis of its lateral branch before wrapping around the superior part of the phlebectasia, passing anteriorly to the phlebectasia to the infrahyoid muscles. The surrounding structures were of normal course and position, but were compressed by the IJV dilatation. No significant variations were observed in the veins on the left side or the superficial right veins. No macroscopic 
pathologies were also observed in the neck region. The medical history of the cadaver was not available.

\section{DISCUSSION}

The IJV is an important vascular structure for oncologists and radiologists and is also a frequently used central venous route. The divisions and phlebectasias of the IJV are quite rare anatomical variations. The concomitant occurrence of both entities has only been documented in two prior reports revealed during diagnostic imaging $[8,9]$. In both reports, internal jugular phlebectasia involved one branch of the duplicated or fenestrated IJV while in the case presented the phlebectasia involved nonfenestrated segment of the IJV.

The IJV was fenestrated in the superior part of the carotid triangle. The window between the medial and lateral branches of the IJV fenestration was wide and no structure passed through it. The inferior - nonfenestrated segment of the IJV had large dilatation. Som et al. [9] documented a case of the right side division of the IJV, the anterior branch of which was dilated in the lower neck. Since both branches united before the termination of the IJV in the right subclavian vein, the division was misdiagnosed as duplication instead of fenestration [4]. In case reported by Rossi and Tortori-Donati [8] the right IJV was duplicated and the phlebectasia involved the terminal segment of the medial branch. A concurrent partial duplication (fenestration) of the proximal left IJV was presented.

In the case presented, as in two other reported cases $[8,9]$ of concomitant vein division and phlebectasia, the phlebectasia involved the right side, which was in agreement with the right side predilection reported in the literature [7]. This predilection for the right side may be explained by a larger right inferior jugular bulb, the absence of valves in the right brachiocephalic vein, the larger diameter of the right IJV and the direct continuity of the right IJV with the right brachiocephalic vein [7]. The right IJV valves are placed at a higher level than their left-sided counterparts. The valves play an important role in preventing retrograde blood flow. Any increase in intrathoracic pressure is easily and consistently transmitted to the right jugular bulb by the presence of the aforementioned anatomical variations [7].

In the case presented, the phlebectasia was large $(4 \times 7 \mathrm{~cm})$. The average reported size of an internal jugular phlebectasia is $2.5 \times 4.5 \mathrm{~cm}$ [1]. The diameter of the IJV can triple in size during straining $[1,14]$.
In the case presented, the surrounding anatomical structures were visibly compressed, pointing to possible pressure symptoms during life which could have been potentiated during exertion.

The clinical incidence of IJV divisions (duplications or fenestrations) is estimated to be as much as $0.4 \%$ of the population; these cases are mostly unilateral in nature [6]. No data regarding the clinical incidence of internal jugular phlebectasia are available since only sporadic reports have been reported.

The exact cause of phlebectasia still remains unclear [7]. The main assumptions were that it was an idiopathic [12], congenital defect within the muscular layer of the venous wall [14], a mechanical obstruction in the lower neck or upper mediastinum [2], or a compression of the IJV on the right side, between the lung cupula and the clavicle [5]. Internal jugular phlebectasia has also been reported to manifest after prolonged positive pressure ventilation [10]. Therefore, an increase in intrathoracic pressure does indeed play a role in aetiology.

The ontogenesis of IJV fenestrations is unclear and several theories have been proposed. According to vascular theory, the fenestration results from inadequate condensation of the embryonic capillary plexus. In the case presented, vascular theory is the most likely explanation for IJV fenestration. Concomitant phlebectasia points to congenital anomalies of the venous wall. This disease is most frequently reported in children $[8,14]$, and the history of its onset among adults often dates back to childhood. This fact may support the contention that an underlying congenital variant is responsible for the condition [8].

The IJV with double and single segments raises the possibility of the potential for deep venous thrombus formation secondary to changes in flow velocities. Phlebectasia additionally increases the risk of emboli or plaque formation due to slowing of the blood flow and stasis in the widened segment of the vessel. Other complications of the internal jugular phlebectasia are Horner's syndrome, difficulties during swallowing and cessation of voice [10]. These could be attributed to the pressuring effect on the neighbouring structures such as the oesophagus, cervical sympathetic nervous system, as well as the lower cranial nerves within the carotid sheath, especially the vagus including its recurrent laryngeal branch.

While the IJV divisions are usually subclinical [13], internal jugular phlebectasia presents itself clinically 
as a subcutaneous swelling in the anterolateral region of the neck that characteristically increases in size with straining, after a Valsalva manoeuvre has been carried out, coughing, sneezing, crying and decreases at rest or with manual compression [2]. Eventual additional signs and symptoms include: dysphagia [11], a change in voice, painful swelling, slight dyspnoea and a venous hum due to the turbulent flow in the dilated vein segment [2].

It is widely accepted that the phlebectasia should be managed conservatively unless complications occur to avoid air embolism, venous thrombosis, and injury to adjacent important structures in the neck during dissection [7].

\section{CONCLUSIONS}

The presence of the reported anomaly may have serious implications for radiologic examinations and surgical procedures in head and neck regions. Head and neck surgeons, radiologists and intensive care practitioners should be made aware of this rare anomaly to prevent inadvertent injury.

\section{Acknowledgements}

We would like to thank Ivan Blažinovič for his dissection work and Marko Slak for his figure labelling. The work was supported by the Slovenian Research Agency (P3-0043).

\section{REFERENCES}

1. Al-Dousary S. Internal jugular phlebectasia. Int J Pediatr Otorhinolaryngol. 1997; 38(3): 273-280, indexed in Pubmed: 9051432.

2. Bowdler DA, Singh SD. Internal jugular phlebectasia. Int J Pediatr Otorhinolaryngol. 1986; 12(2): 165-171, indexed in Pubmed: 3570682.

3. Cvetko E. Unilateral fenestration of the internal jugular vein: a case report. Surg Radiol Anat. 2015; 37(7):
875-877, doi: 10.1007/s00276-015-1431-x, indexed in Pubmed: 25875636.

4. Downie SA, Schalop L, Mazurek JN, et al. Bilateral duplicated internal jugular veins: case study and literature review. Clin Anat. 2007; 20(3): 260-266, doi: 10.1002/ ca.20366, indexed in Pubmed: 16838288.

5. Garrow E, Kirschtein M, Som ML. Internal jugular phlebectasia: case report and review of the literature. Am J Surg. 1964; 108: 380-383, indexed in Pubmed: 14215119.

6. Nayak BS. Surgically important variations of the jugular veins. Clin Anat. 2006; 19(6): 544-546, doi: 10.1002/ ca.20268, indexed in Pubmed: 16372344.

7. Paleri V, Gopalakrishnan S. Jugular phlebectasia: theory of pathogenesis and review of literature. Int J Pediatr Otorhinolaryngol. 2001; 57(2): 155-159, indexed in Pubmed: 11165654.

8. Rossi A, Tortori-Donati P. Internal jugular vein phlebectasia and duplication: case report with magnetic resonance angiography features. Pediatr Radiol. 2001; 31(2): 134, doi: 10.1007/s002470000350, indexed in Pubmed: 11214685.

9. Som PM, Shugar JM, Sacher M, et al. Internal jugular vein phlebectasia and duplication: CT features. J Comput Assist Tomogr. 1985; 9(2): 390-392, indexed in Pubmed: 3973172.

10. Spiro SA, Coccaro SF, Bogucki E. Aneurysm of the internal jugular vein manifesting after prolonged positive pressure ventilation. Head Neck. 1991; 13(5): 450-452, indexed in Pubmed: 1938366.

11. Sundaram J, Menon P, Thingnum SKS, et al. Dysphagia because of unilateral internal jugular vein phlebectasia in an infant. J Pediatr Surg. 2016; 51(7): 1216-1219, doi: 10.1016/j.jpedsurg.2016.04.019, indexed in Pubmed: 27233370.

12. Walsh RM, Lannigan FJ, McGlashan JA, et al. Jugular bulb phlebectasia. Int J Pediatr Otorhinolaryngol. 1993; 25(1-3): 249-254, indexed in Pubmed: 8436472.

13. Wong BY, Strachan DR, Loney EL. Duplication of internal jugular veins: case report. J Laryngol Otol. 2010; 124(3): 341-344, doi: 10.1017/S0022215109990855, indexed in Pubmed: 19689842.

14. Yokomori K, Kubo K, Kanamori Y, et al. Internal jugular phlebectasia in two siblings: manometric and histopathologic studies of the pathogenesis. J Pediatr Surg. 1990; 25(7): 762-765, indexed in Pubmed: 2199660. 